\title{
Disseminated Herpes Zoster in a Patient with Acute Lymphoblastic Leukaemia: A case study
}

\author{
Shah $\mathrm{MS}^{1}$, Begum $\mathrm{M}^{1}$, Rahman $\mathrm{M}^{1}$, Baqi SMA ${ }^{1}$ \\ ${ }^{1}$ Bangabandhu Sheikh Mujib Medical University, Dhaka, Bangladesh.
}

\section{Introduction}

Varicella zoster virus (VZV) is a member of Herpesviridae family. It can cause two distinct clinical entities: varicella (chickenpox) and herpes zoster (shingles). ${ }^{1,2}$ Herpes Zoster (HZ) is painful, vesicular rash in a limited area on one side of body due to the reactivation of latent VZV in dorsal root ganglia. Involvement of three or more dermatomes is known as disseminated zoster and seen in immunocompromised individuals. It can occur at any age. The risk of herpes zoster increases with old age and in patients with reduced cell mediated immunity such as haematological malignancies, immunosuppressive therapies, HIV infection and transplant recipients. The incidence of herpes zoster has been variably reported as $2 \%$ in chronic myeloid leukaemia (CML), 13\% in chronic lymphocytic leukemia (CLL) and $30 \%$ in transplant recipients. ${ }^{3-5}$ This was a rare case of disseminated herpes zoster in a patient with Acute lymphoblastic leukaemia developed while on chemotherapy progressing to prolonged myelosuppression.

Keywords: Herpes zoster (HZ), Acute lymphoblstic leukemia (ALL), Varicella zoster virus(VZV)

\section{Case Report}

In 2017, a 20 year old male was admitted to Haematology department of Bangabandhu Sheikh Mujib Medical University, Dhaka, Bangladesh with B-cell acute lymphoblastic Leukaemia (BALL) with aberrant expression of CD13 on the basis of clinical features, CBC, PBF, Bone marrow morphology, cytochemistry and immunophenotyping. He was started with hyper CVAD protocol and subsequently had completed 6 cycles which were uneventful and was in complete remission. He developed prolonged cytopenia following $7^{\text {th }}$ cycle of chemotherapy. On day $28^{\text {th }}$ (19 th $^{\text {th }}$ October 2017), he suddenly developed malaise, body ache, fever, generalized maculopapular rash evolved rapidly into vesicular eruption. On examination, the patient was febrile with no clinical evidence of systemic inflammatory response syndrome (SIRS). He had vesicles and pustules with crusting in left groin, upper medial and lateral part of both thighs, both gluteal regions, lower back, chest, abdomen and both upper limbs. Lesions were very painful with intense itching. Palms and soles were spared and there was no lymphadenopathy. Other systemic examinations were normal. CBC, peripheral blood smear, routine biochemistry, liver function test, renal function test, urine examination and chest $\mathrm{x}$ ray were normal. Serology for HIV, Hepatitis A, $\mathrm{B}, \mathrm{C}$ were negative. Blood and urine culture and sensitivity were negative. Patient denied for skin biopsy. Immediately intravenous acyclovir along with antibiotic, opiodesandtricyclic antidepressants were started with the clinical diagnosis of disseminated herpes.

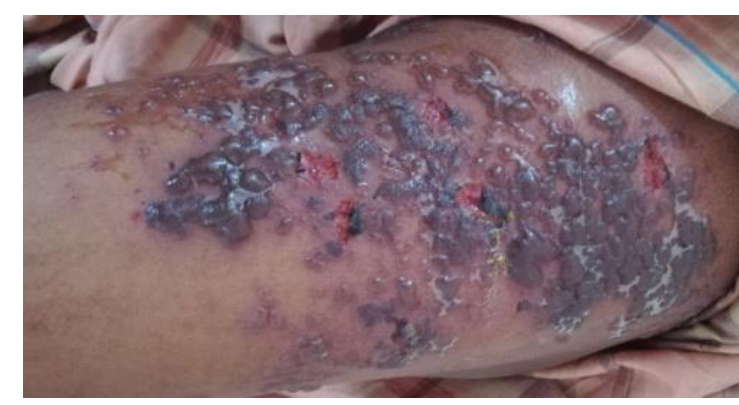

Figure 1: Multiple grouped vesicles, pustular and crusted lesions over left thigh 


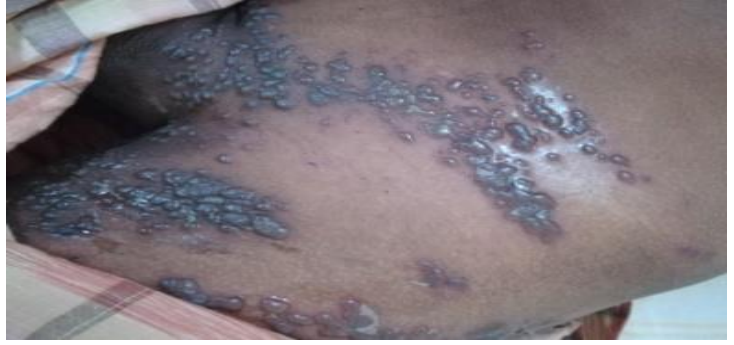

Figure 2: Multiple grouped vesicles, pustular and crusted lesions over left groin and upper thigh

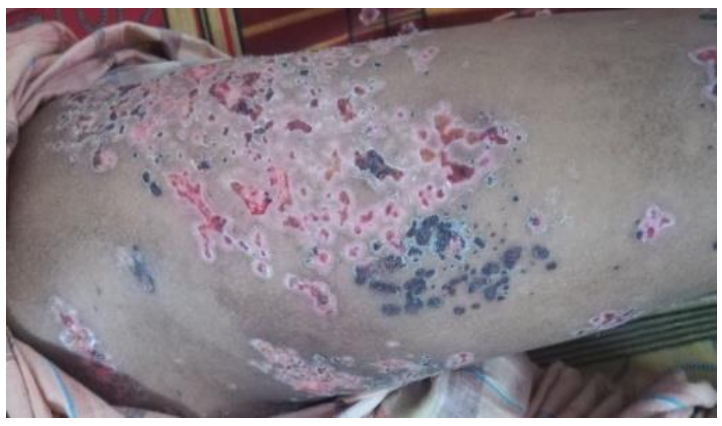

Figure 3: Resolving lesions two weeks of anti-viral therapy

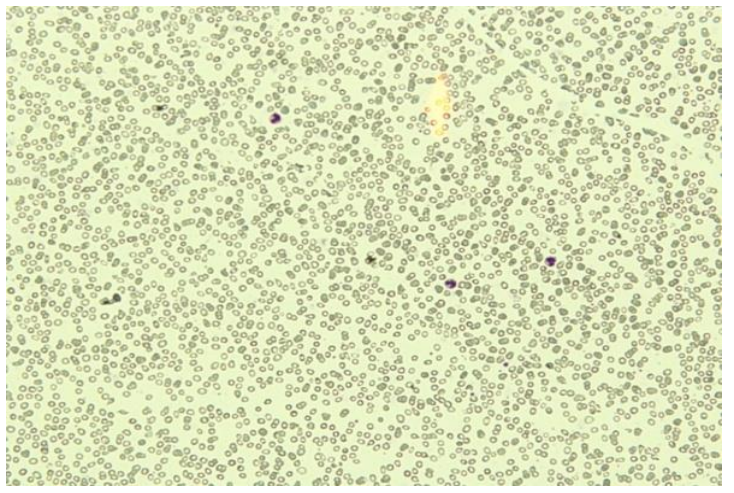

Figure 4: PBF (Low power field) showing pancytopenia on day 39 of chemotherapy

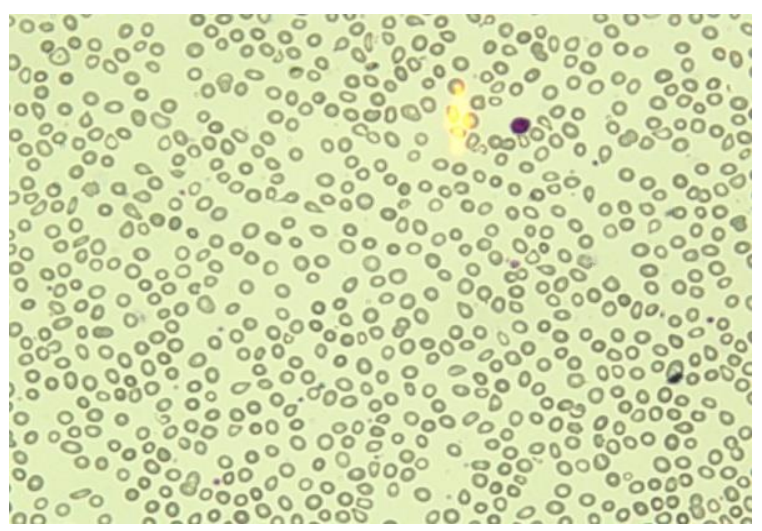

Figure 5: PBF (High power field) showing pancytopenia on day 39 of chemotherapy

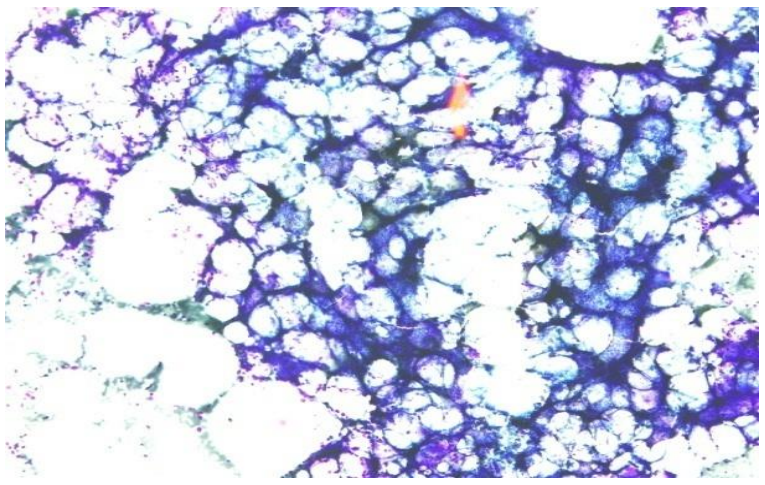

Figure 6: Bone marrow morphology (Low power field) showing hypoplastic marrow on day 39 of chemotherapy

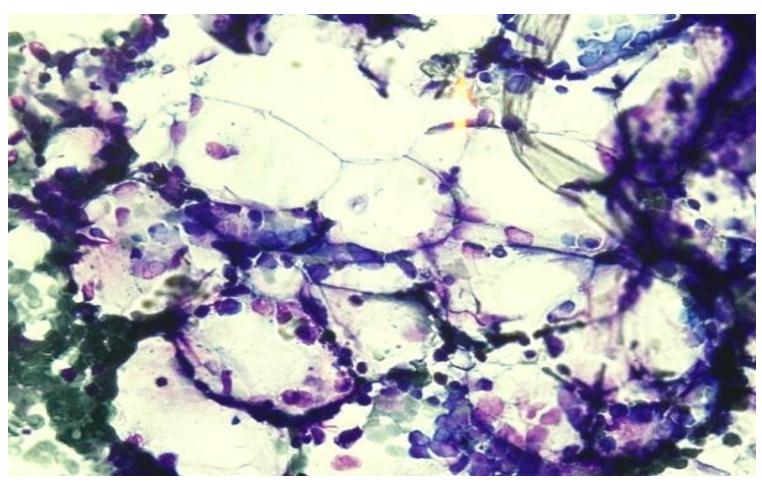

Figure 7: Bone marrow morphology (High power field) showing hypoplastic marrow on day 39 of chemotherapy

On day $39^{\text {th }}$ of the $7^{\text {th }}$ cycle hyper CVAD bone marrow study was done due to persistent cytopenia despite of G-CSF which revealed hypocellular marrow. His lesions resolved over a period of 2 weeks, however, neuralgia was persisted. There were no pneumonitis, meningoencephalitis, hepatitis, and other serious complications. On the day $46^{\text {th }}$ of Chemotherapy, his peripheral blood count was recovered. Varicella zoster virus IgM $(1.79 \mathrm{U} / \mathrm{ml}$, reference value $<8.00 \mathrm{U} / \mathrm{ml})$ was negative and $\mathrm{IgG}(107.98$ $\mathrm{U} / \mathrm{ml}$, Reference range $<8.00 \mathrm{U} / \mathrm{ml}$ ) was positive which were tested in India from a reliable lab through outsourcing, after 3 months of clinical presentation. Initially VZV antibodies, viral culture and PCR for VZV could not be done due to economic constraint and unavailability of these tests facility locally.

\section{Discussion}

Herpes zoster, also known as shingles, and is caused by the reactivation of the varicella-zoster virus. The reactivation is associated with compromised immunity due to condition such as 
haematological malignancy (Leukaemia, lymphoma, multiple myeloma, CLL, CML), diabetes mellitus, autoimmune disease, human immunedeficiency virus (HIV) infection, recepient of transplant and immunosuppressive medications. The incidence of $\mathrm{HZ}$ is up to 15 times higher in HIV-infected patients and it is found in $25 \%$ of patients with Hodgkins lymphoma. ${ }^{6}$ The overall incidence of herpes zoster in patients with Non Hodgkins Lymphoma was $12.21 \% ; 11.79 \%$ of the patients received conventional chemotherapy and $12.76 \%$ of the patients received Rituximab containing chemotherapy. ${ }^{7}$ Herpes zoster occured in $25 \%$ of a population of children with acute lymphoblastic leukaemia (ALL) who were seropositive for varicella zoster virus antibody before its onset; $16.5 \%$ of the total group of children with ALL developed HZ. ${ }^{8} \mathrm{HZ}$ can occur at any age but most commonly affects the elderly population. When the rashes become more widespread and affect three or more dermatomes then it is called disseminated herpes zoster. ${ }^{6}$ Disseminated herpes zoster is less common and generally occurs only in people with compromised or suppressed immune system. This patient presented with characterstic skin finding of disseminated $\mathrm{HZ}$ that was found to be pancytopenic. In this patient, underlying leukemia and chemotherapy induced immunosuppresion could have contributed to dissemination of herpes zoster. It may be associated with encephalitis, pneumonitis, ocular complications and persistent post herpetic neuralgia. But there was no visceral involvement.

The diagnosis of $\mathrm{HZ}$ is usually made clinically on the basis of the characteristic appearance of the $\mathrm{rash}^{9}$. Although shell vial viral culture remains the criterion standard test with which other diagnostic tests are compared, detection of viral DNA by polymerase chain reaction, when available, is the most useful test because it is sensitive and specific and results can be obtained within a few hours. Other tests that can be used to support the diagnosis are direct fluorescent antibody staining, immunoperoxidase staining, histopathology and Tzanck smear. ${ }^{10}$ This patient was diagnosed clinically on the basis of the characteristic appearance of the rash and responded well to intravenous acyclovir. Other tests were not available in this setting to diagnose.

The treatment of choice for disseminated zoster is intravenous acyclovir $10 \mathrm{mg} / \mathrm{kg}$ every 8 hourly for 5-7 days. ${ }^{11}$

Recurrent herpes zoster is exceedingly rare except in immunocompromised hosts, especially those with AIDS.

Varicella may be severe and even fatal in immunocompromised patients: a $30 \%$ rate of dissemination with a $7 \%$ fatality rate has been reported in leukemic children in whom VZV infection developed in the era of effective antiviral therapies.

\section{Conclusion}

Disseminated herpes zoster is a potentially serious infection that can present in immunocompromised patients. Any vesicular rash in immunocompromised patients should be evaluated for herpes zoster. Early diagnosis and aggressive treatment with intravenous acyclovir can reduce morbidity and severity of complications.

\section{References}

1. Kasper D, Fauci A, Hauser S, Longo D, Jameson J, Loscalzo J. Harrison's principles of internal medicine. 19ed. USA 2015. p 1183-85.

2. Kumar V, Abbas AK, Aster JC. Robbins and Cotran Pathologic Basis of Disease. $9^{\text {th }}$ ed. Elsevier Health Sciences. 2014. p 358-59

3. Keating MJ, O'Brien S, Lerner S et al. Long-term follow-up of patients with chronic lymphocytic leukemia(CLL) receiving fludarabine regimens as initial therapy. Blood.1998; 92:1165-71.

4. Mattiuuzi GN, Cortes JE, Talpaz M, et al. Development of varicella zoster virus infection in patients with chronic myelogenous leukemia treated with imatinib mesylate. Clic Cancer Res. 2003;9: 97680 .

5. Locksley RM, Flournoy N, Sulvian KM, et al. Infection with varicella zoster virus fter marrow transplantation. J Infect Dis. 1985;152:1172-81.

6. Nair Preeti, HarshkantGharote, Pooja Singh, et al. Herpes zoster on the face in the elderly. BMJ Case Rep. 2014

7. Shih-Feng Cho, Wan-Hsuan Wu, Yi-Hsin Yang, et al. Longitudinal risk of herpes zoster in patients with 
non-Hodgkin lymphoma receiving chemotherapy: A nationwide population-based study. Sci Rep. 2015;

8. Novelli VM, Brunell PA, Geiser CF, Narkewicz S, Frierson L. Herpes zoster in children with acute lymphocytic leukemia. American Journal of Diseases of Children. 1988; 142:71-72.

9. Sampathkumar P, Drage LA, Martin DP. Herpes zoster (shingles) and postherpetic neuralgia. In Mayo Clinic Proceedings. 2009 Mar 1 (Vol. 84, No. 3, pp. 274-80). Elsevier.
10. Espy MJ, Teo R, Ross TK et al. Diagnosis of varicella zoster virus infection in the clinical laboratory by Lighyder PCR. J Clin Microbiol. 2000; 38:3187-3189.

11. Gupta S, Jain A, Gardiner C, Tyring SK. A rare case of disseminated cutaneous zoster in an immunocompetent patient. BMC Family Practice. 2005; 6: 50

*Correspondence: Md. Salahuddin Shah, Department of Hematology, Bangabandhu Sheikh Mujib Medical University, Dhaka, Bangladesh. e-mail: shah.fcps@gmail.com 